\title{
Progress toward Monte Carlo - Thermal Hydraulic Coupling using Low-Order Nonlinear Diffusion Acceleration Methods
}

\author{
Bryan R. Herman*, Benoit Forget, Kord Smith \\ Massachusetts Institute of Technology, 77 Massachusetts Ave., Cambridge, MA 02139
}

\begin{abstract}
A new approach for coupled Monte Carlo (MC) and thermal hydraulics (TH) simulations is proposed using low-order nonlinear diffusion acceleration methods. This approach uses new features such as coarse mesh finite difference diffusion (CMFD), multipole representation for fuel temperature feedback on microscopic cross sections, and support vector machine learning algorithms (SVM) for iterations between CMFD and TH equations. The multipole representation method showed small differences of about $0.3 \%$ root mean square (RMS) error in converged assembly source distribution compared to a conventional MC simulation with ACE data at the same temperature. This is within two standard deviations of average real uncertainty. Eigenvalue differences were on the order of $10 \mathrm{pcm}$. Support vector machine regression was performed on-the-fly during MC simulations. Regression results of macroscopic cross sections parametrized by coolant density and fuel temperature were successful and eliminated the need of partial derivative tables generated
\end{abstract}

\footnotetext{
${ }^{*}$ Corresponding author

Email addresses: bherman@mit.edu (Bryan R. Herman), bforget@mit.edu (Benoit Forget), kord@mit.edu (Kord Smith)

Preprint submitted to Annals of Nuclear Energy

October 20, 2014
} 
from lattice codes. All of these new tools were integrated together to perform MC-CMFD-TH-SVM iterations. Results showed that inner iterations between CMFD-TH-SVM are needed to obtain a stable solution.

Keywords: Monte Carlo, thermal hydraulics, OpenMC, CMFD, support vector machines, Multipole representation, BEAVRS

\section{Introduction}

One of the many goals in reactor analysis is to predict accurate spatial power distributions. Currently, the industry standard is to perform simulations using nodal diffusion methods accelerated by nonlinear diffusion acceleration (NDA) methods such as coarse mesh finite difference (CMFD). These nodal methods rely on homogenized multigroup diffusion parameters (cross sections, discontinuity factors, etc.) generated from separate lattice calculations. During homogenization, all detailed information is lost and can only be recovered using reconstruction methods at the end of simulation. Thus, refinement in space and energy is not possible to achieve a more accurate solution. Nodal methods are commonly coupled to low-order thermal hydraulic (TH) methods to treat reactivity feedback through fuel temperature (Doppler), coolant density, etc. Commonly, a full nodal solution is generated, thermal hydraulic fields such as fuel temperature and coolant density are calculated, cross section interpolation is performed and the process repeats. This paper addresses coupling fuel temperature and coolant density fields to a higher order continuous-energy Monte Carlo solution.

Monte Carlo methods are attractive for reactor analysis because multigroup cross section generation is not required. These methods use point-wise 
in energy cross section data. In addition, geometry can be explicitly modeled using constructive solid geometry. This class of methods can provide accurate pin-wise power distributions because tallies can be scored on any size mesh given that enough neutron histories are simulated. Because individual neutrons are simulated to obtain results, these methods are notoriously slow. Also, the stochastic process of sampling probability distributions requires that statistical uncertainty be accumulated and reported. Monte Carlo methods are different in the fact that after a converged source is obtained, tallies for spatial power distributions are then collected.

In this paper, progress toward a different approach is investigated where thermal hydraulic fields are converged during the source convergence stage of Monte Carlo through a low-order CMFD operator. A new on-the-fly Doppler broadening technique called windowed multipole representation is used for fuel temperature feedback (Forget et al., 2014; Josey et al., 2014). Different acceleration methods are explored using machine learning techniques to determine how CMFD parameters depend on TH parameters.

For the analyses presented in this work, the OpenMC Monte Carlo code is used (Romano and Forget, 2013). Section 2 provides a background of how Monte Carlo methods are used for analyzing reactors. Section 3 discusses CMFD and its implementation into OpenMC. The thermal hydraulic model incorporated into the Monte Carlo code is presented in Section 4, and the coupling between neutronic and thermal hydraulic fields is described in Section 5. Finally, simulations performed and results are discussed in Sections 6 and 7 , respectively. 


\section{Monte Carlo simulations for reactor analysis}

Monte Carlo methods for reactor analysis are commonly referred to as eigenvalue or criticality simulations. These methods differ from fixed source analyses because the neutron source is unknown and depends on the whole system. Thus, eigenvalue simulations are separated into two parts: 1) inactive source iterations to converge the spatial distribution of the fission source, and 2) active source iterations where tallies are accumulated.

During inactive fission source generations, an initially assumed source is iterated on until it is converged analogous to power iteration. Neutrons are born from source sites contained in a finite source bank and simulated until they escape the domain or are absorbed. As these neutrons are simulated they can cause fission where a new site will be stored in the next source bank. The number of inactive fission source generations is determined by the user and convergence is commonly assessed with Shannon entropy (Brown, 2006). This diagnostic produces a scalar value for the spatial distribution of the fission source. Once it is stationary about some mean, the source is determined to be converged. The number of neutrons stored in the source bank can also be controlled by the user and is referred to as number of neutron histories per generation. Because we may tally CMFD quantities during inactive fission source generations, each generation will also be a tally batch. Thus, inactive fission source generations are the same as inactive batches.

Once a source is converged, tally means and variances are accumulated. To reduce bias, the source bank is updated at the end of each fission source generation. Because source sites used in a generation are directly dependent 
on fission sites from the previous generation, generations are highly correlated. This tends to produce inaccurate estimates of variances and confidence intervals (Brissenden and Garlick, 1986; Ueki, 2010). Kelly et.al. have shown that to reduce this inaccuracy, users can run multiple fission source generations in a tally batch (Kelly et al., 2012). All of these choices that users can make affects the behavior of MC tally and source convergence. The terminology presented in this section will be referred to throughout this paper.

\section{CMFD diffusion acceleration}

Coarse mesh finite difference diffusion methods were developed decades ago and are now commonly used to accelerate fission source convergence in nodal simulations (Smith, 1983). This nonlinear iteration strategy has been proven to reduce both computational time and storage when used to accelerate nodal methods. Recently, CMFD has been applied to eigenvalue Monte Carlo simulations to accelerate fission source convergence (Lee et al., 2012). When CMFD acceleration takes place, tallies need to be present during inactive batches. The most important equation that needs to be satisfied by MC tallies, to within stochastic uncertainty, is the neutron balance equation. This equation is written for a uniform Cartesian CMFD mesh with dimensions $\left(\Delta_{l}^{u}, \Delta_{m}^{v}, \Delta_{n}^{w}\right)$ as 


$$
\begin{aligned}
& \sum_{u \in(x, y, z)}\left\langle\bar{J}_{l+1 / 2, m, n}^{u, g} \Delta_{m}^{v} \Delta_{n}^{w}\right\rangle-\left\langle\bar{J}_{l-1 / 2, m, n}^{u, g} \Delta_{m}^{v} \Delta_{n}^{w}\right\rangle \\
& +\left\langle\overline{\bar{\Sigma}}_{l, m, n}^{g} \overline{\bar{\phi}}_{l, m, n}^{g} \Delta_{l}^{u} \Delta_{m}^{v} \Delta_{n}^{w}\right\rangle=\sum_{h=1}^{G}\left\langle{\overline{\overline{\nu_{s} \Sigma}}}_{s_{l, m, n} \rightarrow g}^{\left.h \rightarrow \bar{\phi}_{l, m, n}^{h} \Delta_{l}^{u} \Delta_{m}^{v} \Delta_{n}^{w}\right\rangle}\right. \\
& +\frac{1}{k} \sum_{h=1}^{G}\left\langle{\overline{\overline{\nu_{f} \Sigma}}}_{f_{l, m, n} \rightarrow g}^{\left.h \rightarrow \bar{\phi}_{l, m, n}^{h} \Delta_{l}^{u} \Delta_{m}^{v} \Delta_{n}^{w}\right\rangle .}\right.
\end{aligned}
$$

The parameters in brackets $\langle\cdot\rangle$ in Eq. (1) are quantities that can be tallied in MC. Each tally is described below:

- $\left\langle\bar{J}_{l \pm 1 / 2, m, n}^{u, g} \Delta_{m}^{v} \Delta_{n}^{w}\right\rangle$ - surface area integrated net current over surface at $(l \pm 1 / 2, m, n)$ with surface normal in direction $u$ in energy group $g$. By dividing this quantity by the transverse area, $\Delta_{m}^{v} \Delta_{n}^{w}$, the surface area averaged net current can be computed.

- $\left\langle\overline{\bar{\Sigma}}_{t, m, n}^{g} \overline{\bar{\phi}}_{l, m, n}^{g} \Delta_{l}^{u} \Delta_{m}^{v} \Delta_{n}^{w}\right\rangle$ — volume integrated total reaction rate over energy group $g$.

- $\left\langle{\overline{\overline{\nu_{s} \Sigma}}}_{s_{l, m, n}}^{h \rightarrow g} \overline{\bar{\phi}}_{l, m, n}^{h} \Delta_{l}^{u} \Delta_{m}^{v} \Delta_{n}^{w}\right\rangle$ — volume integrated scattering production rate of neutrons that begins with energy in group $h$ and exits reaction in group $g$. This reaction rate also includes the energy transfer of reactions (except fission) that produce multiple neutrons such as $(\mathrm{n}, 2 \mathrm{n})$; hence, the need for $\nu_{s}$ to represent neutron multiplicity.

- $k$ - core multiplication factor.

- $\left\langle{\overline{\overline{\nu_{f} \Sigma}}}_{f_{l, m, n}}^{h \rightarrow g} \overline{\bar{\phi}}_{l, m, n}^{h} \Delta_{l}^{u} \Delta_{m}^{v} \Delta_{n}^{w}\right\rangle$ — volume integrated fission production rate of neutrons that begins with energy in group $h$ and exits in group $g$. 


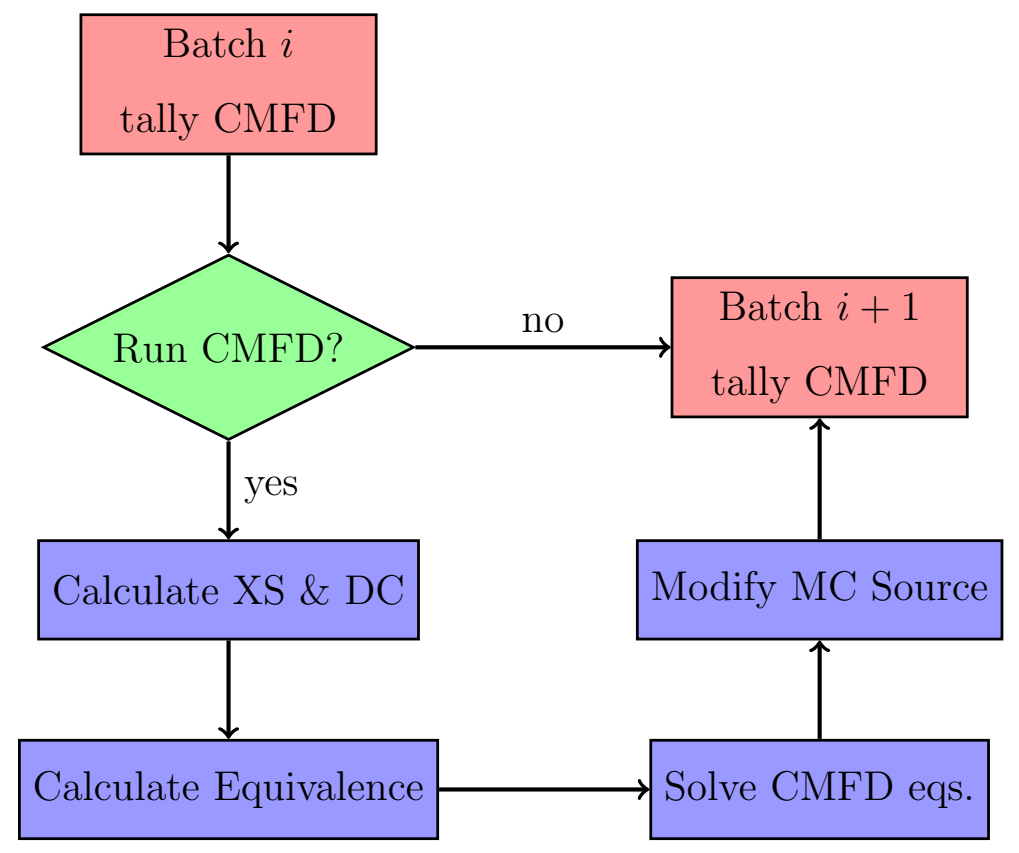

Figure 1: Flow chart of CMFD execution between tally batches. 
MC tallies will satisfy this equation to within stochastic uncertainty as tally batches are accumulated. CMFD parameters can be calculated from this equation and a better fission source can be obtained. An outline of CMFD accelerated MC is presented in Figure 1. After each batch of neutrons, CMFD feedback may be applied before simulating the next batch of neutrons. The details of each part of CMFD are detailed in the following subsections.

\subsection{Calculation of macroscopic cross sections and diffusion coefficients}

Results from MC tallies are used to compute macroscopic cross sections (XS) and diffusion coefficients (DC) that are needed to solve the multigroup diffusion equation. In Eq. (1), macroscopic cross sections are already detailed out for each reaction rate. Macroscopic total, scattering production and fission production are obtained respectively with:

$$
\begin{aligned}
\overline{\bar{\Sigma}}_{t, m, n}^{g} & =\frac{\left\langle\overline{\bar{\Sigma}}_{t_{l, m, n}}^{g} \overline{\bar{\phi}}_{l, m, n}^{g} \Delta_{l}^{u} \Delta_{m}^{v} \Delta_{n}^{w}\right\rangle}{\left\langle\overline{\bar{\phi}}_{l, m, n}^{g} \Delta_{l}^{u} \Delta_{m}^{v} \Delta_{n}^{w}\right\rangle}, \\
{\overline{\overline{\nu_{s} \Sigma}}}_{s_{l, m, n}}^{h \rightarrow g}= & \frac{\left\langle{\overline{\overline{\nu_{s} \Sigma}}}_{s_{l, m, n} \rightarrow g}^{\left.h \rightarrow \bar{\phi}_{l, m, n}^{h} \Delta_{l}^{u} \Delta_{m}^{v} \Delta_{n}^{w}\right\rangle}\right.}{\left\langle\overline{\bar{\phi}}_{l, m, n}^{h} \Delta_{l}^{u} \Delta_{m}^{v} \Delta_{n}^{w}\right\rangle}
\end{aligned}
$$

and

$$
{\overline{\overline{\nu_{f} \Sigma}}}_{f_{l, m, n}}^{h \rightarrow g}=\frac{\left\langle{\overline{\overline{\nu_{f} \Sigma}}}_{f_{l, m, n} \rightarrow g}^{\left.h \rightarrow \bar{\phi}_{l, m, n}^{h} \Delta_{l}^{u} \Delta_{m}^{v} \Delta_{n}^{w}\right\rangle}\right.}{\left\langle\overline{\bar{\phi}}_{l, m, n}^{h} \Delta_{l}^{u} \Delta_{m}^{v} \Delta_{n}^{w}\right\rangle} .
$$

The numerator and denominator in each of these equations is a single tally bin in MC. Diffusion coefficients are needed to relate net current and scalar flux in the diffusion equation. Because equivalence between diffusion theory 
and transport theory is performed in the next step, the perfect diffusion coefficient does not need to be obtained. However, better diffusion coefficients will lead to faster convergence and a more stable acceleration. Diffusion coefficients are difficult to calculate in MC because a single volume-average diffusion coefficient cannot describe the leakage out of each surface of a cell. In addition, because we are using a coarse mesh, significant spatial truncation error exists. Besides the previous issues, more accurate diffusion coefficient suited for light water reactor (LWR) applications can be obtained by performing an in-scatter correction on anisotropic scattering isotopes and performing an energy collapse from a fine distribution of diffusion coefficients to a few groups (Herman et al., 2013b). The calculation of diffusion coefficients in this work is as follows:

$$
\overline{\bar{D}}_{l, m, n}^{g}=\frac{\left\langle\overline{\bar{\phi}}_{l, m, n}^{g} \Delta_{l}^{u} \Delta_{m}^{v} \Delta_{n}^{w}\right\rangle}{3\left\langle\overline{\bar{\Sigma}}_{t r_{l, m, n}}^{g} \overline{\bar{\phi}}_{l, m, n}^{g} \Delta_{l}^{u} \Delta_{m}^{v} \Delta_{n}^{w}\right\rangle},
$$

where

$$
\begin{aligned}
\left\langle\overline{\bar{\Sigma}}_{t r_{l, m, n}}^{g} \overline{\bar{\phi}}_{l, m, n}^{g} \Delta_{l}^{u} \Delta_{m}^{v} \Delta_{n}^{w}\right\rangle & =\left\langle\overline{\bar{\Sigma}}_{t_{l, m, n}}^{g} \overline{\bar{\phi}}_{l, m, n}^{g} \Delta_{l}^{u} \Delta_{m}^{v} \Delta_{n}^{w}\right\rangle \\
& -\left\langle{\overline{\overline{\nu_{s} \Sigma}}}_{s 1_{l, m, n}}^{g} \overline{\bar{\phi}}_{l, m, n}^{g} \Delta_{l}^{u} \Delta_{m}^{v} \Delta_{n}^{w}\right\rangle .
\end{aligned}
$$

Note that in order to obtain the transport reaction rate, the total reaction rate is reduced by the $P_{1}$ scattering reaction rate.

\subsection{Calculation of equivalence parameters}

Using a second order finite volume discretization, surface area averaged net current can be related to volume averaged scalar flux with

$$
\bar{J}_{l+1 / 2, m, n}^{u, g}=-\tilde{D}_{l+1 / 2, m, n}^{g}\left(\overline{\bar{\phi}}_{l+1, m, n}^{g}-\overline{\bar{\phi}}_{l, m, n}^{g}\right)
$$


Equation (7) represents an interface between two cells. The parameter $\tilde{D}_{l+1 / 2, m, n}^{g}$ is a function of diffusion coefficients and cell dimensions. These relationships are also derived for various boundary conditions. Equation (7) can be combined with Eq. (1) to obtain a diffusion equation. This diffusion equation is inconsistent with $\mathrm{MC}$ transport for many reasons. The first is that leakage between cells, characterized by a diffusion coefficient, is not accurate. Another reason is spatial truncation error that would result in the solution because a coarse mesh is being used. To make the diffusion equation consistent with the transport solution, leakage is preserved between cells using a modified form of Eq. (7),

$$
\begin{aligned}
\bar{J}_{l+1 / 2, m, n}^{u, g} & =-\tilde{D}_{l+1 / 2, m, n}^{g}\left(\overline{\bar{\phi}}_{l+1, m, n}^{g}-\overline{\bar{\phi}}_{l, m, n}^{g}\right) \\
& +\hat{D}_{l+1 / 2, m, n}^{g}\left(\overline{\bar{\phi}}_{l+1, m, n}^{g}+\overline{\bar{\phi}}_{l, m, n}^{g}\right)
\end{aligned}
$$

The parameter $\hat{D}_{l+1 / 2, m, n}^{g}$ is calculated for every surface and energy group by solving Eq. (8). All other parameters in this equation can be derived from MC tallies.

If the neutron balance equation is satisfied by MC tallies on a coarse mesh, reaction rates from this equation can be conserved by computing macroscopic cross sections. Leakage rates can also be conserved by fixing the inconsistencies from spatial truncation error and diffusion approximations with an extra parameter that is derived from $\mathrm{MC}$ tallies. Note, this only provides accurate averaged values over the coarse mesh to which it is applied. 


\subsection{CMFD equations}

Combining Eqs. (8) and (1), a linear system of equations can be created for every spatial cell and energy group. These form a generalized eigenvalue problem that has the form

$$
\begin{aligned}
& \sum_{u \in x, y, x} \frac{1}{\Delta_{l}^{u}}\left[\left(-\tilde{D}_{l-1 / 2, m, n}^{u, g}-\hat{D}_{l-1 / 2, m, n}^{u, g}\right) \overline{\bar{\phi}}_{l-1, m, n}^{g}\right. \\
& +\left(\tilde{D}_{l-1 / 2, m, n}^{u, g}+\tilde{D}_{l+1 / 2, m, n}^{u, g}-\hat{D}_{l-1 / 2, m, n}^{u, g}+\hat{D}_{l+1 / 2, m, n}^{u, g}\right) \overline{\bar{\phi}}_{l, m, n}^{g} \\
& \left.+\left(-\tilde{D}_{l+1 / 2, m, n}^{u, g}+\hat{D}_{l+1 / 2, m, n}^{u, g}\right) \overline{\bar{\phi}}_{l+1, m, n}^{g}\right] \\
& +\overline{\bar{\Sigma}}_{t_{l, m, n}}^{g} \overline{\bar{\phi}}_{l, m, n}^{g}-\sum_{h=1}^{G}{\overline{\overline{\nu_{s} \Sigma}}}_{s_{l, m, n}}^{h \rightarrow g} \overline{\bar{\phi}}_{l, m, n}^{h}=\frac{1}{k} \sum_{h=1}^{G}{\overline{\overline{\nu_{f} \Sigma}}}_{f_{l, m, n}}^{h \rightarrow g} \overline{\bar{\phi}}_{l, m, n}^{h} .
\end{aligned}
$$

Standard generalized eigenvalue solvers are used to solve this system such as power iteration and Jacobian-free Newton Krylov methods.

At this step, the CMFD system of equations can also be used for other applications. Herman et al. (2013a) presented results where this set of equations was used to compute adjoint distributions, higher harmonic distributions and dominance ratio. Because these equations have consistent physics with the transport solution, many applications are possible.

\subsection{Modification of Monte Carlo source bank}

Once a multigroup flux distribution is obtained from solving the transport consistent diffusion equations, a normalized source distribution can be calculated. This normalized source distribution represents the probability of a source neutron being born in a given coarse mesh cell and energy group. 
This is obtained with

$$
p_{l, m, n}^{g}=\frac{\sum_{h=1}^{G}{\overline{\overline{\nu_{f} \Sigma}}}_{f_{l, m, n}}^{h \rightarrow g} \overline{\bar{\phi}}_{l, m, n}^{h} \Delta_{l}^{u} \Delta_{m}^{v} \Delta_{n}^{w}}{\sum_{n} \sum_{m} \sum_{l} \sum_{h=1}^{G}{\overline{\overline{\nu_{f} \Sigma}}}_{f_{l, m, n}}^{h \rightarrow g} \overline{\bar{\phi}}_{l, m, n}^{h} \Delta_{l}^{u} \Delta_{m}^{v} \Delta_{n}^{w}} .
$$

By multiplying this equation by the total number of neutrons simulated in a batch, the expected number of neutrons born in a spatial cell and energy band is determined. The ratio of this quantity and the actual number of neutrons in the MC source bank in each corresponding cell and energy band can be calculated. This is shown as

$$
f_{l, m, n}^{g}=\frac{N p_{l, m, n}^{g}}{\sum_{i} w_{i}} ; \quad i \in(g, l, m, n),
$$

where $N$ is the total number of neutrons simulated in a batch, $w_{i}$ is the weight of neutrons in a corresponding energy group and spatial cell, and $f_{l, m, n}^{g}$ are termed weight adjustment factors. If the distributions agree exactly, these factors are unity. Otherwise, weights of neutrons in the MC source bank are modified by this multiplicative factor,

$$
w_{i}^{\prime}=w_{i} \times f_{l, m, n}^{g} ; \quad i \in(g, l, m, n) .
$$

\section{Thermal hydraulics}

For the TH model, simplifications are made to obtain a simple set of equations because we are only interested in studying the coupling effect. Future work will include using more complex models. The following assumptions are made in the $\mathrm{TH}$ model:

1. Single-phase fully developed flow, 


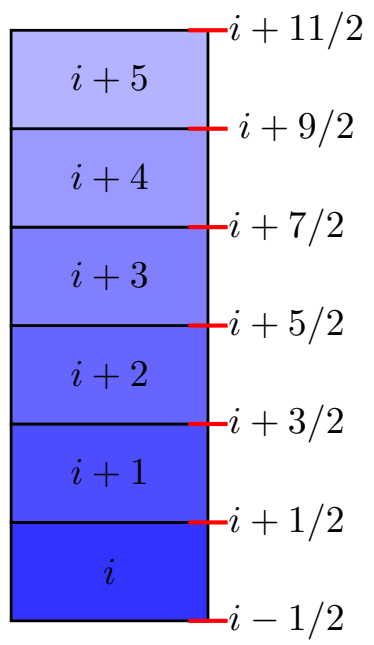

Figure 2: Diagram of TH domain.

2. Infinite mixing of coolant in an assembly and no cross flow between assemblies,

3. Known flow rate into each assembly and pressure is fixed in each channel,

4. Fission energy deposition is local to volume and can be approximated by fission neutron production rate,

5. Bundle averaged quantities are calculated.

Due to these assumptions, only an energy conservation equation is needed to determine spatial distribution of coolant density. A simple diagram is presented in Figure 2 to show how the energy equation is solved. Domain $i$ is used as an example. The inlet enthalpy at $i-1 / 2$, the energy deposited within $i$ and flow rate of coolant are known. The outgoing enthalpy is determined from an energy balance with

$$
h_{i+1 / 2}=h_{i-1 / 2}+\frac{\dot{q}_{i}}{\dot{m}} .
$$


The inlet and exit enthalpy of a cell can be used to calculate a cell averaged enthalpy. Here, we take a simple average. The average enthalpy and pressure is used in the equation of state for water to determine density.

The second quantity of interest is the average fuel temperature. A simple conduction equation is applied between the coolant, clad, gap and fuel to determine average fuel temperature. It is calculated with the following formula:

$$
\bar{T}_{f}=T_{m}+\frac{q^{\prime}}{2 \pi}\left[\frac{1}{4 k_{f}}+\frac{1}{R_{g} h_{g}}+\frac{1}{k_{c}} \ln \left(\frac{R_{c o}}{R_{c i}}\right)+\frac{1}{R_{c o} h_{m}}\right],
$$

where

- $\bar{T}_{f}$ is the average fuel temperature,

- $T_{m}$ is the temperature of water in the cell determined from equation of state,

- $q^{\prime}$ is the linear heat rate,

- $k_{f}$ is the fuel conductivity,

- $R_{g}$ is the mean gap radius calculated by taking the average of clad inner radius and fuel pellet radius,

- $h_{g}$ is the gap conductivity,

- $k_{c}$ is the conductivity of clad,

- $R_{c o}$ and $R_{c i}$ are outer and inner clad radius, respectively,

- and $h_{m}$ is the coolant heat transfer coefficient. 
The linear heat rate is calculated by dividing cell power by the height of the cell. Geometry information is discussed in Section 6. The fuel conductivity and clad conductivity are $2.4 \mathrm{~W} / \mathrm{m}-\mathrm{K}$ and $17 \mathrm{~W} / \mathrm{m}-\mathrm{K}$, respectively. The gap conductivity is held constant at $31000 \frac{\mathrm{W}}{\mathrm{m}^{2}-\mathrm{K}}$. The only parameter that needs to be calculated is the heat transfer coefficient of water. This is performed using the Dittus-Boelter correlation (Todreas and Kazimi, 2010).

\section{Neutronic - TH coupling}

Coupling $\mathrm{MC}$ neutronics data to $\mathrm{TH}$ is a rather straightforward process assuming that a tally mesh is placed over the MC geometry that is on the same nodalization for TH physics. During the MC simulation, energy deposition tallies are accumulated (approximated by fission production rate) and are then normalized to produce power peaking factors over that mesh. Then, local power in each cell can be computed by multiplying average cell power with its power peaking factor. This is then used directly in the energy equation presented in Eq. (13).

The TH physics produces a spatial distribution of coolant density and fuel temperature over the tally mesh. To feedback coolant density to MC, number densities of coolant nuclides must be recalculated. To have a unique water material for coolant density feedback in each assembly, water material objects are replicated in the OpenMC materials input file. Another option for this feedback is to change the number densities on the fly by finding the cell in which the particle is currently in, every time water cross sections are needed. Fuel temperature changes affect microscopic cross sections through Doppler broadening. There are several different methods that 
have been used to account for varying fuel temperature and are described by Vazquez (Vazquez et al., 2012). These include loading explicit cross sections at a certain temperature interval, performing on-the-fly Doppler broadening and mixing cross sections of different temperatures with a certain weighted average. In this work, the multipole representation method is used to perform on-the-fly Doppler broadening for U-235 and U-238 in the resolved resonance range and is discussed in Section 5.2.

The conventional method for coupling neutronic and $\mathrm{TH}$ together is to run a full MC simulation, compute thermal hydraulics and then rerun MC simulation again. Increased computational efficiency can be achieved by not running fully converged MC simulations until later iterations and also starting from the previously converged source distribution. This is illustrated in Fig. 3, except TH feedback is performed between batches. In this paper, a different approach is used where MC, CMFD and TH will converge together during inactive fission source generations. Because CMFD is being used to accelerate source convergence, CMFD-TH iterations can be fully converged when acceleration is applied. This process is highlighted in Figure 4. This will be compared to $\mathrm{MC}$ directly coupled to $\mathrm{TH}$ during inactive fission source generations presented in Figure 3. However, because CMFD-TH iterations are now considered, macroscopic cross sections and diffusion coefficients that are used in CMFD equations are dependent on coolant density and fuel temperature. For deterministic transport/diffusion codes and multigroup MC codes, this is provided through given functional relationships or lookup tables generated from lattice calculations. In continuous energy MC, we want to directly use ACE libraries and, therefore, are not able to precompute these 


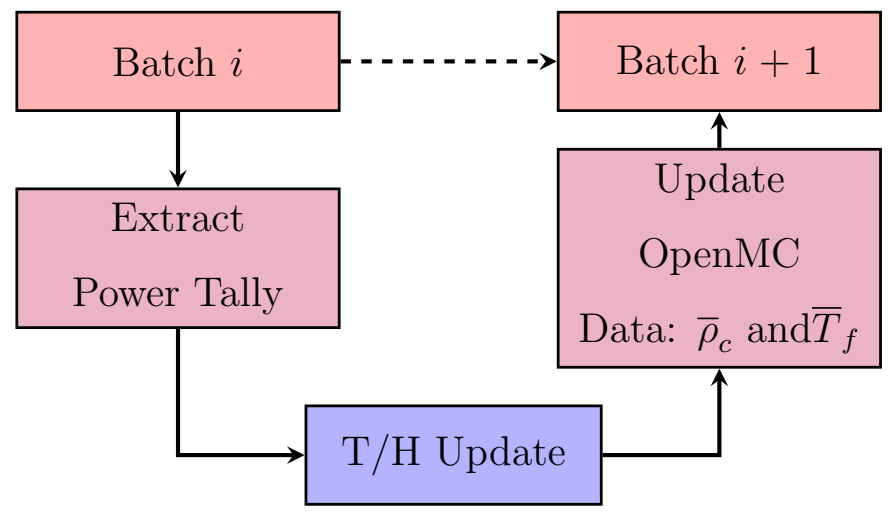

Figure 3: Conventional MC-TH coupling except feedback is applied during fission source iterations.

relationships. To overcome this challenge, Machine Learning techniques are used to learn these dependencies during MC simulations. This is reflected by the Train SVR with XS and Predict XS with SVR blocks in Figure 4. This is discussed more thoroughly in the next section.

\subsection{Support vector machines for $X S$ regression}

Support Vector Machines (SVM) is a machine learning method that is widely used for classification (SVC) and regression (SVR) (Chistianini and Shawe-Taylor, 2000). This section will provide a high level view of SVMs. This type of machine learning is supervised where training data are provided before predictions are made. Training data are given to SVR tools in the form $\left\{\left(\vec{x}_{1}, y_{1}\right), \ldots,\left(\vec{x}_{n}, y_{n}\right)\right\}$, where $\vec{x}$ is a feature vector that describes a label $y$. In the context of TH feedback, a feature vector may include information such as fuel temperature and coolant density, and a label may be a macroscopic cross section. 


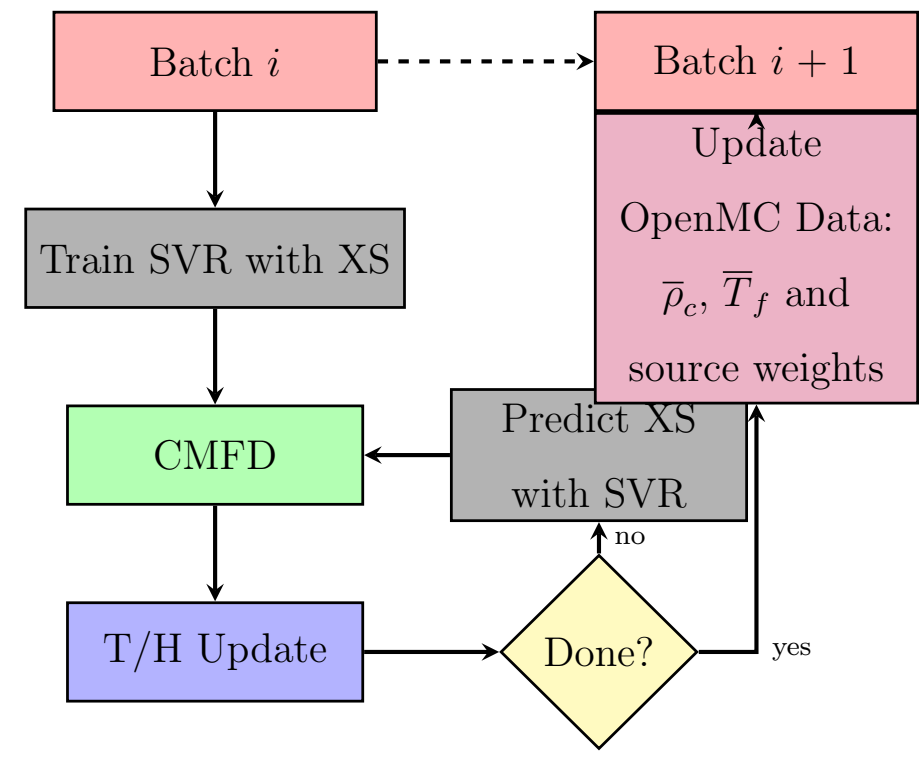

Figure 4: Proposed new coupling methodolgy where inner CMFD-TH iterations are converged between MC fission source iterations.

The easiest regression to perform is linear. A linear function in SVR is represented as

$$
f(\vec{x})=\langle\vec{w}, \vec{x}\rangle+b,
$$

where $\vec{w}$ is a vector of weights and $b$ is the offset from the origin. The goal of this process is to minimize the norm of the weight vector. Cortes and Vapnik formulated the following optimization problem (Cortes and Vapnik, 1995):

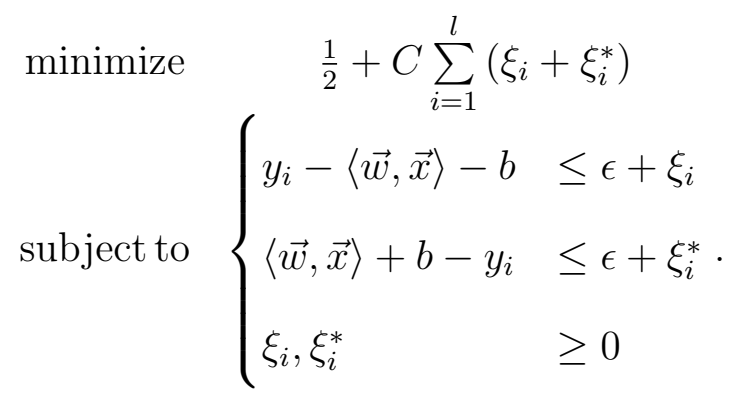




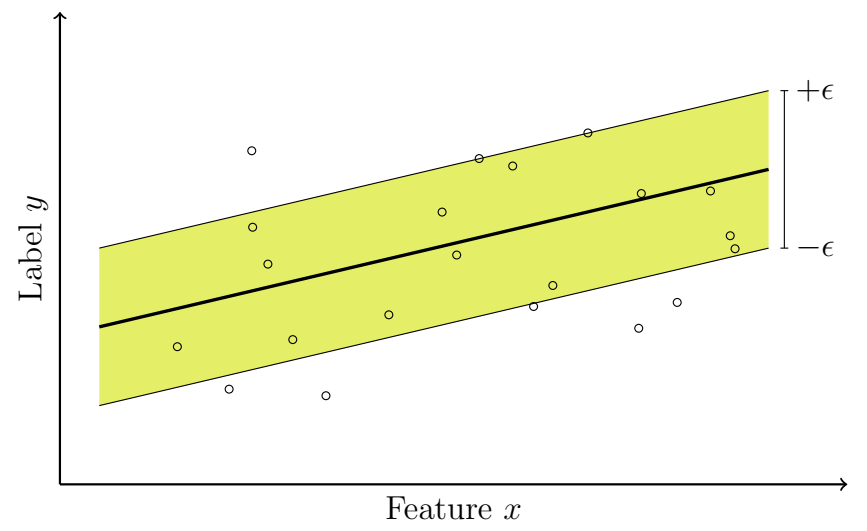

Figure 5: $\epsilon$-insensitive loss function in linear SVR.

In Equation (16), $\xi_{i}$ and $\xi_{i}^{*}$ are defined as slack variables that help with constraints of the optimization problem and improve generalization. The parameter $C$ is introduced as a regularization parameter which penalizes more complex models to avoid over-fitting the training data. The parameter $\epsilon$ is used in the $\epsilon$-insensitive loss function where values outside of $\pm \epsilon$ are penalized. Figure 5 depicts this type of loss function where only points outside of the shaded $\pm \epsilon$ region contribute to the loss. For nonlinear algorithms, feature vectors are mapped to higher dimensional feature spaces, represented as $\vec{x} \rightarrow \Phi(\vec{x})$, using kernels. A nice trick with kernels is that the explicit mapping of the feature vector to the feature space, $\Phi(\vec{x})$, is not required. Rather, only an implicit mapping is required because SVM algorithms only depend on inner products of feature vectors. A kernel is therefore defined as $K\left(\vec{x}_{i}, \vec{x}_{j}\right) \equiv\left\langle\Phi\left(\vec{x}_{i}\right), \Phi\left(\vec{x}_{i}\right)\right\rangle$. The kernel function used in this work is the Gaussian radial basis function, formulated as

$$
K\left(\vec{x}_{i}, \vec{x}_{j}\right)=\exp \left(-\gamma\left\|\vec{x}_{i}-\vec{x}_{j}\right\|^{2}\right)
$$


where $\gamma$ is a free parameter commonly taken as the inverse of the number of features. The capability of nonlinear regression will be very important in TH feedback, especially when combined effects of TH parameters, spectral effects and depletion are considered.

For each cross section, a separate SVR problem is solved. The specifics of feature vectors and labels will be discussed in Section 6. Once each SVR problem is trained, a prediction can be made for what the change in cross section should be. A cross section change is preferred because the MC simulation already provides a good estimate of the cross-section at the previous conditions. As an example, if both coolant density and fuel temperature are independent $\mathrm{TH}$ parameters used in regression, a new cross section at $\mathrm{TH}$ iteration $i$ can be calculated with

$$
\Sigma^{i}\left(T_{f}^{i}, \rho^{i}\right)=\Sigma^{0}\left(T_{f}^{0}, \rho^{0}\right)+\frac{\partial \Sigma}{\partial T_{f}}\left(T_{f}^{i}-T_{f}^{0}\right)+\frac{\partial \Sigma}{\partial \rho}\left(\rho^{i}-\rho^{0}\right),
$$

where $\Sigma$ represents a macroscopic cross section, $T_{f}$ is fuel temperature, $\rho$ is coolant density, $i$ is current thermal iteration and 0 represents reference conditions at which partial derivatives were computed. Equation (18) shows a simple multidimensional linear interpolation where each $\mathrm{TH}$ parameter is considered as a separate impact. This assumption is adequate because dependencies on TH parameters are performed separately in single assembly lattice calculation. However, when the same type of understanding is being performed on-the-fly with a full core model, nonlinear terms will impact cross section values. To account for this, when macroscopic cross section feedback is performed for CMFD diffusion iterations, the following feedback model is 
used:

$$
\begin{aligned}
\Sigma_{M C}^{i}\left(T_{f}^{i}, \rho^{i}\right) & =\Sigma_{M C}^{0}\left(T_{f}^{0}, \rho^{0}\right) \\
& +\left[\Sigma_{S V R}^{i}\left(T_{f}^{i}, \rho^{i}\right)-\Sigma_{S V R}^{0}\left(T_{f}^{0}, \rho^{0}\right)\right] .
\end{aligned}
$$

In Eq. (19), $\Sigma_{M C}$ is the cross section computed from MC tallies and $\Sigma_{S V R}$ is the cross section evaluated by the SVR problem. Reference TH conditions are coolant density and fuel temperature distributions used during the $\mathrm{MC}$ simulation. All nonlinear effects are accounted for in the difference between what SVR thinks the cross section should be at the new TH conditions and the cross sections at the reference TH conditions, which might differ slightly from the MC result.

\subsection{Multipole method for fuel temperature feedback}

One of the most difficult parts in coupling TH to MC neutronics is how to take into account how Doppler broadening affects microscopic cross sections. The brute-force approach is to load cross section libraries at a wide range of temperatures and perform interpolation on-the-fly. This approach was studied by Trumbull (2006) and concluded thattens to hundreds of gigabytes would be required. Over the past decade, other on-the-fly Doppler broadening techniques have been created that reduce this storage size requirement to tens of gigabytes (Vitanen and Leppänen, 2012; Yesilyurt et al., 2012). Recently, Forget et.al. studied and implemented the multipole representation method to perform on-the-fly Doppler broadening which further reduce the storage size to 100s of megabytes (Forget et al., 2014).

Forget implemented this approach in OpenMC for the resolved energy range of U-235 and U-238. Results were compared between point-wise cross 
section data sets at a specific temperature and the multipole representation approach at that same temperature. Comparisons yielded good agreement between the two methods with a slight increase in computational cost of about $10 \%$. Recent work has focused on optimizing the windowed multipole method that would eliminate the computational increase (Josey et al., 2014).

In the results presented in this paper, the multipole method was used to perform fuel temperature feedback. This was performed specifically for $\mathrm{U}-235$ and $\mathrm{U}-238$ in the resolved energy range. Outside this energy range, cross sections are taken from standard point-wise ACE files at one specified temperature.

\section{Description of simulations}

OpenMC will be used to perform all Monte Carlo simulations. CMFD and TH solvers have been embedded into OpenMC. The code is linked to LIBSVM through a custom Fortran/C++ interface to perform SVM calculations (Chang and Lin, 2011). To test various new components of the proposed methodology, a 2-D version of the MIT BEAVRS benchmark is used. Specifications of the benchmark are described in Reference (Horelik et al., 2013). To create the $2-\mathrm{D}$ model, a radial slice was taken at $225.0 \mathrm{~cm}$. A diagram of this radial slice is shown in Figure 6. There are no grid spacers or control rods present in this model and all instrument tubes are replaced with guide tubes such that the core is quarter symmetric. Although the core is symmetric, a full azimuthal model is used in the MC simulations. This is important because it exacerbates dominance ratio effects when converging fission source distributions and other tallies. 


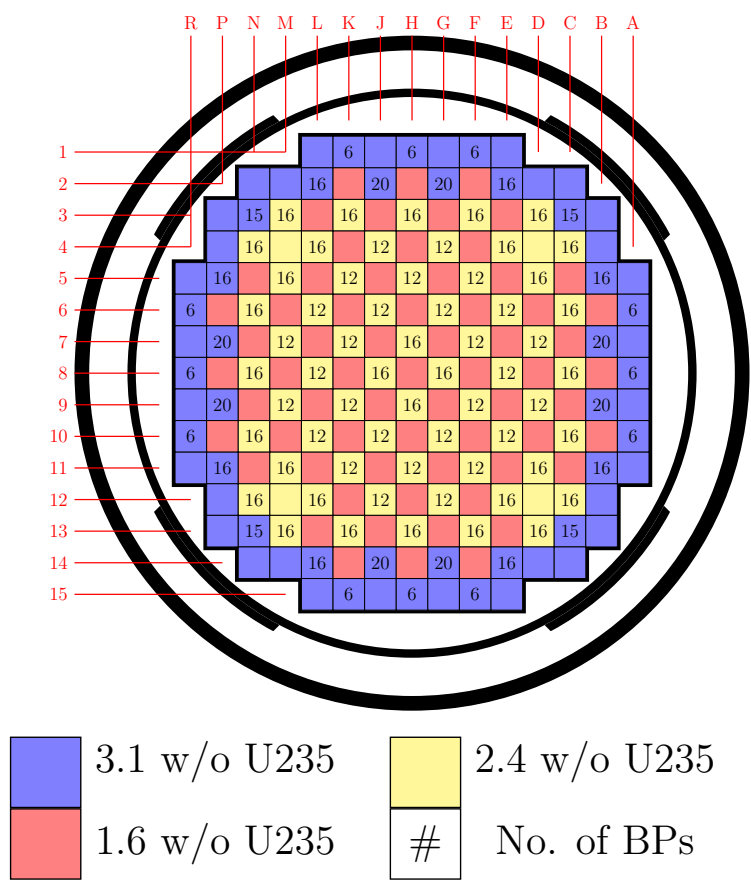

Figure 6: BEAVRS 2-D geometry plot used in simulations. 
An assembly mesh is used both for CMFD acceleration and TH calculations. Diffusion calculations performed in the CMFD methodology will only encompass assemblies and not mesh corners that only contain moderator and structural material. Therefore, an unstructured Cartesian mesh is used where boundary conditions between core/reflector interface are needed. In OpenMC, partial current tallies on mesh surfaces can be accumulated. By allowing partial current tallies instead of net current, albedos (ratio of incoming to outgoing current on a surface) can be calculated for every mesh surface between core and reflector. This is very important to do because mesh cells far away from the core will have very few neutrons contributing to tally bins. This approach circumvents the need for good tally estimates in these peripheral regions.

Instead of starting the system at hot zero power conditions as specified in the benchmark, random distributions of assembly averaged coolant density and fuel temperature are used. This is important when performing SVR, because a large sample space of coolant density and fuel temperature needs to be provided so that SVR can learn trends in cross section data. The following cross sections are used in the regression: (1) fast absorption, $\Sigma_{a}^{1}$, (2) thermal absorption, $\Sigma_{a}^{2}$, (3) effective downscatter, $\hat{\Sigma}_{s}^{1 \rightarrow 2}$, (4) fast fission production, $\nu \Sigma_{f}^{1}$, (5) thermal fission production, $\nu \Sigma_{f}^{2}$, (6) fast transport, $\Sigma_{t r}^{1}$ and (7) thermal transport, $\Sigma_{t r}^{2}$. From these basic cross sections, all CMFD parameters can be calculated. In the core, there are nine types of assemblies, listed below:

1. $1.6 \%$ enrichment no burnable poisons (BPs) - 65 bundles,

2. $2.4 \%$ enrichment no BPs - 4 bundles, 
3. $2.4 \%$ enrichment 12 BPs - 28 bundles,

4. $2.4 \%$ enrichment 16 BPs - 32 bundles,

5. 3.1\% enrichment no BPs - 32 bundles,

6. $3.1 \%$ enrichment 6 BPs - 12 bundles,

7. $3.1 \%$ enrichment 15 BPs - 4 bundles,

8. $3.1 \%$ enrichment 16 BPs -8 bundles,

9. $3.1 \%$ enrichment 20 BPs -8 bundles.

Each of these assemblies can have a different coolant density and fuel temperature combination. Four attributes are selected for each training data: (1) enrichment, (2) number of BPs, (3) coolant density and (4) fuel temperature. Items (1) and (2) are used to classify the bundle type and the last two are TH parameters.

\section{Results}

This section presents results of different components needed to perform integral TH feedback. This includes the performance of CMFD acceleration for neutronic-only simulations, as well as the effect of using multipole representation to account for Doppler broadening of Uranium resonances in the resolved resonance energy range.

\subsection{CMFD acceleration at HZP TH conditions}

The effect of CMFD acceleration on multigroup Monte Carlo source convergence has been studied extensively by Lee et.al (Lee et al., 2012). The results shown here are for accelerating a continuous energy Monte Carlo code 
where point-wise representation of resonances are used. This can make convergence more difficult because more neutrons are needed in the simulation to resolve each resonance. A simulation of a case where no CMFD is compared to a CMFD case. Both simulations start with HZP conditions from an initial uniform spatial source guess using 20 million neutrons per fission source generation.

Source convergence is determined by using the Shannon entropy diagnostic on an assembly mesh. This is one method for determining when the source becomes stationary and better methods to determine convergence are beyond the scope of this analysis. Results are presented in Figure 7. From the plot, it takes approximately 25 batches for the CMFD entropy to become stationary. The entropy where CMFD is not applied does not converge until 150 batches. CMFD looks to converge to about the same Shannon entropy as the base case without CMFD. It will not agree exactly, because there is always uncertainty in the converged fission source distribution. The CMFD entropy trend looks very erratic initially compared to the smooth decrease of a conventional MC case. The first drop in entropy is from performing the first CMFD calculation. This estimate of the source distribution, calculated by CMFD, mispredicts the converged source distribution. However, it quickly rises back after a few CMFD iterations. The other depressions in the entropy result from resetting tally bins to eliminate initial source guess bias. After a few resets, the CMFD entropy settles down to a stationary trend. This convergence is exacerbated because of the initial random distribution of temperature and density. This random distribution is needed because it allows the SVM machine learning tool to explore a wide space of 


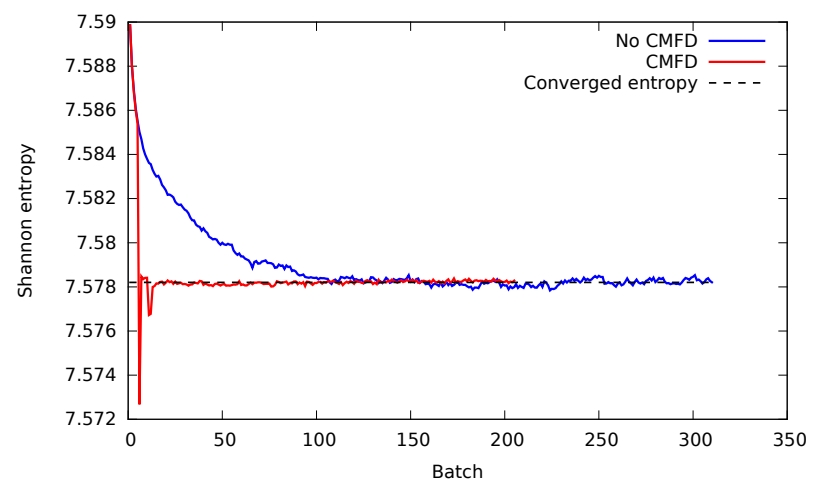

Figure 7: CMFD acceleration of fission source convergence for HZP conditions.

temperatures and densities. Normally, an isothermal starting guess is used and Monte Carlo converges more rapidly. CMFD will also follow this trend and converge more rapidly.

\subsection{Multipole results}

Before using the multipole method for fuel temperature feedback, comparisons are performed against conventional MC. Three cases are performed: (1) conventional MC with ACE libraries at 900K, (2) multipole MC at 900K with ACE libraries at $900 \mathrm{~K}$ and (3) multipole MC at $900 \mathrm{~K}$ and ACE libraries at $600 \mathrm{~K}$. Although the multipole method does not require ACE libraries, these libraries are still required for cross sections outside of the resolved resonance range. Therefore, when TH feedback is performed on-the-fly, cross sections calculated outside of the resolved resonance range will be at the temperature of the ACE library. This issue is studied by comparing cases (2) and (3). Simulations were performed with 20 million neutrons per batch, one fission source generation per batch, 200 inactive fission source generations and 200 


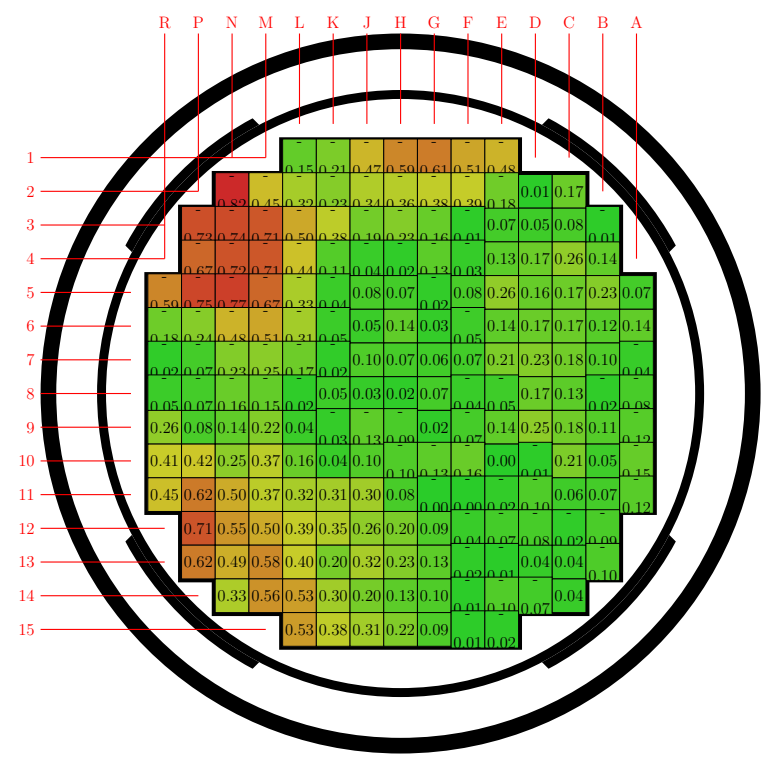

Figure 8: Relative percent error between conventional ACE cross sections and multipole representation method at $900 \mathrm{~K}$.

tally batches. In these cases, a uniform random assembly averaged coolant density distribution was used instead of a constant density.

A comparison of assembly fission source tallies is first performed between cases (1) and (2). These results are presented in Figure 8. Results show that errors are in the tenths of percent with the largest difference being $0.82 \%$ and root mean square error of $0.298 \%$. Another interesting observation is that although the core is symmetrically loaded, error distributions in the source are not. This is caused by high dominance ratio and correlated sampling effects. The eigenvalues of case (1) and case (2) were about $10 \mathrm{pcm}$ different. Case (1) yielded a k-effective of 0.99646(1) and case (2) yielded a k-effective of $0.99658(1)$. Although these are out of the statistical uncertainty range 


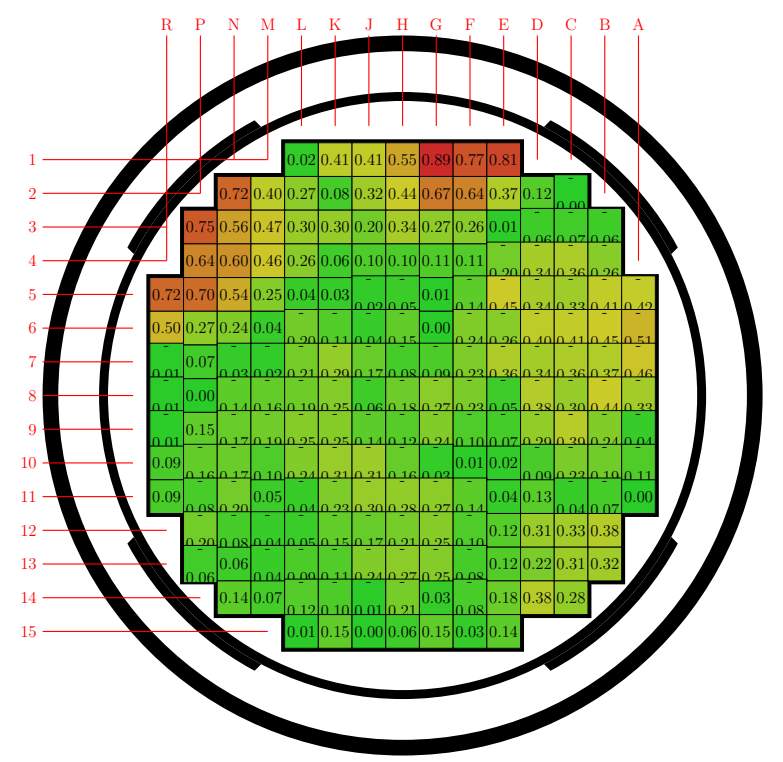

Figure 9: Comparison of fuel temperature effects (relative percent difference) not captured by multipole method outside of resonance range.

of a couple of $\mathrm{pcm}$, the comparison is still good taking into account that a completely different representation of resonances was used.

Cases (2) and (3) were compared and results are presented in Figure 9. Differences observed in this comparison are very similar to the ones observed in the previous comparison. The largest error observed is $0.89 \%$ and root mean square error of $0.288 \%$. The eigenvalue of case (3) is $0.99697(1)$. This is about $40 \mathrm{pcm}$ different than the eigenvalue reported from case (2). Therefore, although we do not see much difference in the source distribution, the eigenvalues show more difference than between cases (1) and (2). This extra difference can be attributed to temperature dependent effects in the fuel outside of the resolved resonance range. This case showed a $300 \mathrm{~K}$ perturbation between multipole and ACE library temperatures. When fuel temperature 


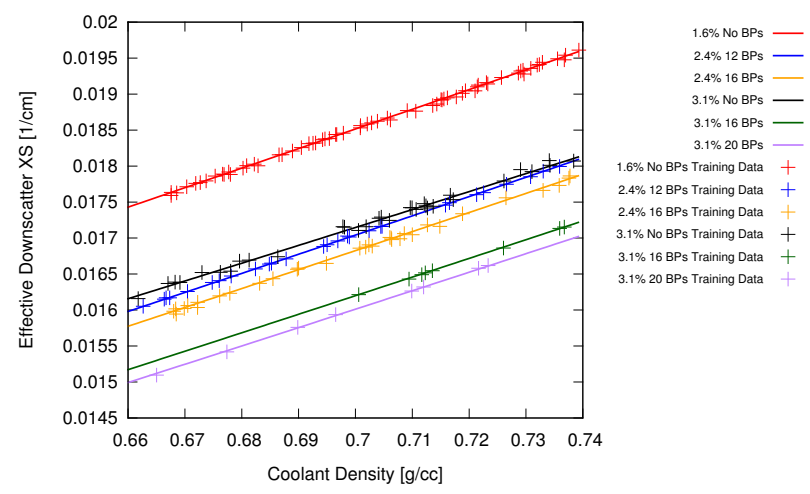

Figure 10: Training and prediction data for coolant density regression of effective downscatter cross section.

feedback is performed, $300 \mathrm{~K}$ deviations are not expected between feedback temperature and default ACE library temperature. Future work will study on-the-fly possibilities for regions outside the resolved resonance range.

\subsection{Support vector regression on cross sections}

This section presents results for three different types of regressions. The first is coolant density, the second is fuel temperature and the last is both TH parameters. The parameters set for SVM regressions are, $C=1.0, \epsilon=$ $1 \times 10^{-6}$ and $\gamma=0.25$ in Eqs. (16) and (17). These parameters were chosen by performing cross-validation tests where $95 \%$ of training was used to train the SVM model and the extra 5\% was used for prediction. Root mean square error values were then computed for an array of the above parameters and the chosen values represented data well. For each regression, a full MC simulation was performed with 20 million neutrons per fission source generation, 200 inactive generations, a single generation per tally batch and 300 tally batches. After tallies were collected, each cross section type was trained in separate 
SVM models from assembly averaged values. The first result presented is a regression test of effective downscatter cross section with density shown in Figure 10. In this figure, six assemblies were chosen to compare prediction regressions and training data. Although data is shown by bundle type, only one SVM model was used to obtain these regression data. Overall, agreement is good between training data and prediction trends. Although training data can be noisy, some of the training data has different spectrum effects which is not represented in the feature vector currently. Even with these effects, the data is linear as expected because coolant density has a direct effect on the magnitude of macroscopic cross sections.

A similar analysis was performed for fuel temperature. In Figure 11, fuel temperature regression data is shown for fast absorption cross section. Most of the trends have a slight bend in them. This is consistent with fuel temperature usually being represented as a square root to achieve a linear trend. Finally, regression on both fuel temperature and coolant density was performed and is presented in Figure 12. The 2-D color map on this plot represents the magnitude of the fast absorption cross section for a given coolant density and fuel temperature. The points plotted in the axial direction represents the absolute difference between training data and prediction of training data using SVM model at a given spot on the 2-D map. Although training and prediction of training data do not need to agree, it is expected that the error is small and no trends exist. The RMS between these data is $0.05 \%$. This analysis shows that SVM machine learning tools implemented into OpenMC are behaving as expected. 


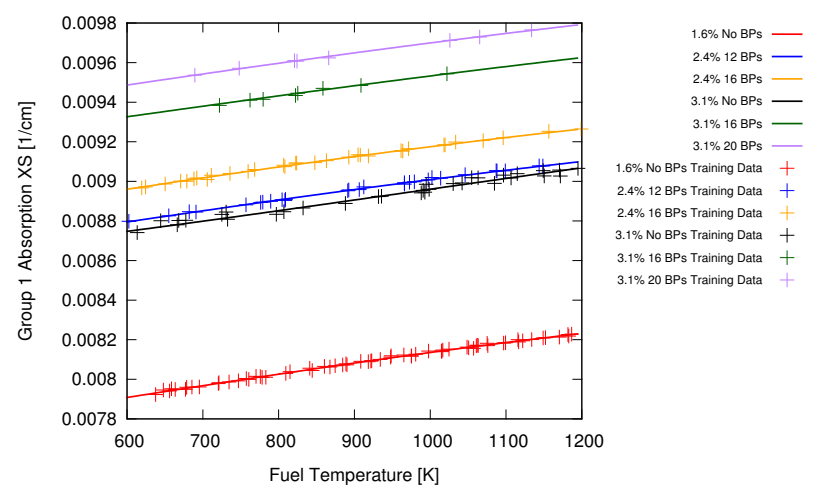

Figure 11: Training and prediction data for fuel temperature regression of fast absorption cross section.

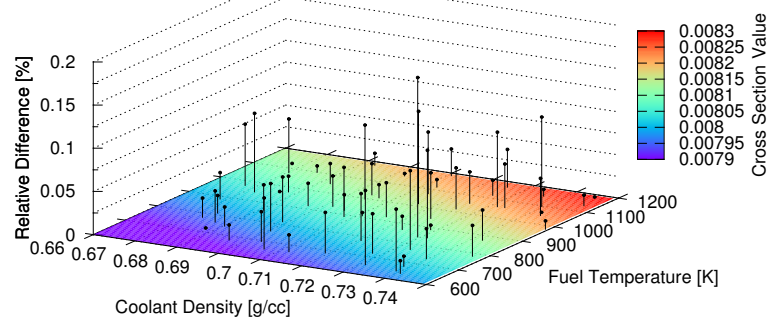

Figure 12: Training and prediction data for fuel temperature and coolant density regression of fast absorption cross section of $1.6 \%$ assembly. 


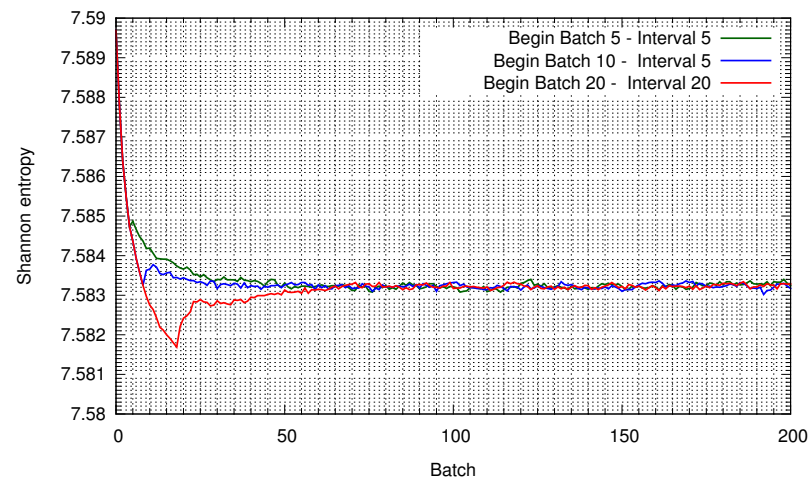

Figure 13: Source convergence for TH feedback using MC source distribution.

\subsection{Thermal hydraulic feedback}

The 2-D BEAVRS model was used to study axially-integrated TH feedback from a process standpoint. Axially-integrated refers to the fact that axial 1-D assembly TH distributions of coolant density and fuel temperature are averaged axially to one parameter per assembly. Results presented in this section show how different TH feedback strategies perform. This information will help when studying full 3-D simulations. The first set of results for TH feedback includes no CMFD diffusion calculations. This method, described by Fig. 3, uses the MC source distribution to compute and update TH parameters. This is very similar to the conventional style of performing MC-TH feedback, except here we perform the feedback process during the convergence of inactive fission source generations. This was performed for 3 running strategies and results are shown in Fig. 13. Each of the curves plotted start TH feedback at different batches. For the runs that began at batch 5 and 10, TH feedback was performed every 5 batches. For the last run that began at batch 20, TH feedback was performed every 20 batches. Different 
initial batch numbers were chosen to verify that the same final entropy was reached independent of what the initial source was that performed the initial TH feedback. This feedback process was performed at two different intervals to study the dependence on the number of tally batches accumulated between feedback stages. Figure 13 shows that all plots converge to the same final entropy and each has different characteristics depending on when they started and how often feedback was applied. Shannon entropy indicates that all simulations converge by batch 65 . These trends converge very nicely and converge much sooner than the initial guess which took 150 batches according to results presented in Figure 7. This is in part because running TH feedback provides a flatter distribution of density and temperature closer to the initial uniform source guess than a fission source with HZP conditions.

The next set of analyses studied the effects of incorporating CMFD into the feedback process. For this study, 4 different simulations were compared:

1. TH feedback using MC source with no CMFD,

2. TH feedback using MC source with CMFD,

3. TH feedback using CMFD source with no CMFD-TH inner iterations,

4. TH feedback using CMFD source with CMFD-TH inner iterations using SVM.

In each case above, TH iterations began at batch 10 and updates were applied at an interval of 10 batches. For the cases 2-4, CMFD began at batch 5. Shannon entropy source convergence results are shown in Fig. 14 for simulations 1, 2, 3 and 4. In addition, because CMFD tallies must be reset periodically because $\mathrm{TH}$ conditions are changing, a moving tally window was implemented. For example, at a certain CMFD iteration tally batches 2-5 


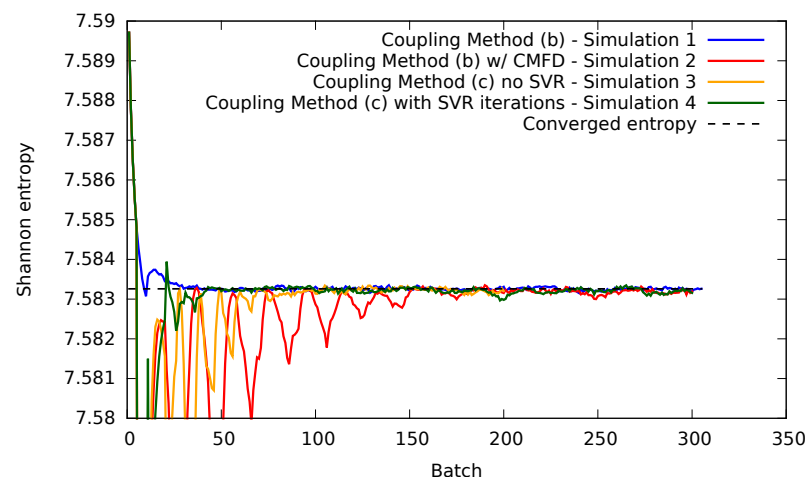

Figure 14: Source convergence for different TH feedback methods.

may be used to compute CMFD cross sections. On the next CMFD iteration, batch 2 is removed and tallies from batches 3-6 are used. The first observation in Fig. 14 is that case 2 and case 3 exhibit oscillatory behavior. This may be due to the fact that MC and $\mathrm{TH}$ are performed one after another and are never fully consistent like in case 4 . However, eventually, these simulations converge to a steady value. One positive result form this study is that inner iterations between CMFD, TH and SVM must be performed when using CMFD. By performing this inner iteration process, more stable convergence behavior was achieved. The next step in the coupling process is to look at convergence rates of fuel temperature and coolant density, not just the MC source distribution. In addition, these simulations need to be extended to full 3-D reactor models.

\section{Conclusions}

In this paper, progress toward TH feedback in MC simulations was discussed. Two methodologies were investigated. The first is similar to con- 
ventional MC-TH iterations, except tallies are accumulated during inactive fission source generations and TH feedback is applied during source convergence. The second is to extend CMFD source acceleration to perform coupled TH iterations on the diffusion level before feeding back coolant density and fuel temperature to MC. Both of these methods showed stable iteration processes where Shannon entropy was used as a metric for source convergence.

In order to perform MC feedback of fuel temperature, the multipole representation method was used. This method allowed cross sections to be reconstructed at any temperature that $\mathrm{TH}$ equations predicted in the resolved resonance range. A study was performed to look at the differences of using the multipole method compared to direct ACE cross sections. Differences of about $0.3 \%$ RMS in source distribution were found and eigenvalue differences on the order of $10 \mathrm{pcm}$. Part of this $0.3 \%$ RMS error is due to statistical uncertainty, as well as cross section representation difference. In order to perform CMFD-TH iterations, the dependence of diffusion parameters on TH fields must be estimated. Because this dependence is not known beforehand, a SVM learning tool was introduced. In order for this tool to learn trends in data, a random distribution of coolant density and fuel temperature was used and training data for machine learning was produced from MC tallies. A study was performed that indicated that SVM could predict trends of macroscopic cross sections with respect to both fuel temperature and coolant density.

Finally, TH iterations were performed with conventional MC source and CMFD source. Conventional MC showed very nice convergence to a stationary source distribution. Inner CMFD-TH iterations were needed because 
a one pass through CMFD-TH equations yielded oscillatory results. More analyses are needed to look at why this instability occurs. Although this paper shows that CMFD-TH-SVM iterations are stable, convergence rates of TH fields must be studied to determine if CMFD accelerates convergence for both source and TH distributions. This work must be extended to full 3-D core simulations to study the impact of convergence when $\mathrm{TH}$ parameters vary axially.

\section{Acknowledgements}

This research was performed under appointment of the first author to the Rickover Fellowship Program in Nuclear Engineering sponsored by Naval Reactor Division of the US Department of Energy.

\section{References}

Brissenden, R., Garlick, A., 1986. Biases in the estimation of keff and its error by Monte Carlo methods. Annals of Nuclear Energy 13, 63-83.

Brown, F., September 10 - 14 2006. On the use of Shannon entropy of the distribution for assessing convergence of Monte Carlo criticality calculations. In: Proceedings of PHYSOR 2006. Vancouver, BC, Canada.

Chang, C.-C., Lin, C.-J., 2011. LIBSVM: A library for sup-
port vector machines. ACM Transactions on Intelligent Sys-
tems and Technology 2, 27:1-27:27, software available at
http://www.csie.ntu.edu.tw/ cjlin/libsvm.


Chistianini, N., Shawe-Taylor, J., 2000. An Introduction to Support Vector Machines and other kernal-based learning methods. Cambridge University Press, Cambridge.

Cortes, C., Vapnik, V., 1995. Support-vector networks. Machine learning 20, 273-297.

Forget, B., Xu, S., Smith, K., 2014. Direct doppler broadening in Monte Carlo simulations using the multipole representation. Annals of Nuclear Energy 64, 78-85.

Herman, B., Forget, B., Smith, K., 2013a. Utilizing CMFD in OpenMC to estimate dominance ratio and adjoint. Transactions of American Nuclear Society 109, 1389-1392.

Herman, B. R., Forget, B., Smith, K., Aviles, B. N., May 5 - 9 2013b. Improved diffusion coefficients generated from Monte Carlo codes. In: Proceedings of M\&C 2013. Sun Valley, ID, USA.

Horelik, N. E., Herman, B. R., Forget, B., Smith, K. S., May 5 - 9 2013. Benchmark for evaluation and validation of reactor simulations (BEAVRS). In: Proceedings of M\&C 2013. Sun Valley, ID, USA.

Josey, C., Forget, B., Smith, K., Sep. 28 - Oct. 3 2014. Efficiency and accuracy evaluation of the windowed multipole direct doppler broadening method. In: Proceedings of PHYSOR 2014. Kyoto, Japan.

Kelly, D., Sutton, T., Wilson, S., April 15-20 2012. MC21 analysis of the nuclear energy agency Monte Carlo performance benchmark problem. In: Proceedings of PHYSOR 2012. Knoxville, Tennessee, USA. 
Lee, M. J., Joo, H. G., Lee, D., Smith, K., April 15 - 20 2012. Monte Carlo reactor calculation with substantially reduced number of cycles. In: Proceedings of PHYSOR 2012. Knoxville, Tennessee, USA.

Romano, P. K., Forget, B., 2013. The OpenMC Monte Carlo particle transport code. Annals of Nuclear Energy 51, $274-281$.

Smith, K. S., 1983. Nodal method storage reduction by nonlinear iteration. Transactions of American Nuclear Society 44, 265.

Todreas, N. E., Kazimi, M. S., 2010. Nuclear systems. Vol. 1. CRC Press, Boca Raton.

Trumbull, T. H., 2006. Treatment of nuclear data for transport problems containing detailed temperature distributions. Nuclear Technology 156, 75-86.

Ueki, T., 2010. Standard deviation of local tallies in global Monte Carlo calculation of nuclear reactor core. Journal of Nuclear Science and Technology 47, 739-753.

Vazquez, M., et al., 2012. Coupled neutronics thermal-hydraulics analysis using Monte Carlo and sub-channel codes. Nuclear Engineering and Design 250, 403-411.

Vitanen, T., Leppänen, J., 2012. Explicit treatment of thermal motion in continuous energy Monte Carlo tracking routines. Nuclear Science and Engineering $171,165-173$.

Yesilyurt, G., Martin, W., Brown, F., 2012. On-the-fly doppler broadening for Monte Carlo codes. Nuclear Science and Engineering 171, 239-257. 